\title{
Toxoplasmosis, A Protozoon Disease
}

\author{
Rafia Tabassum* \\ Department of Zoology, University of the Punjab, Lahore, Pakistan \\ *Corresponding Author: Rafia Tabassum, Department of Zoology, Punjab University Lahore, Pakistan.
}

Received: June 20, 2019; Published: July 03, 2019

DOI: 10.31080/ASMI.2019.02.0294

Toxoplasmosis is a wide spread zoonotic infection of warm-blooded animals including humans all over the world. This infection caused by a Protozoon parasite Toxoplasma gondii. The parasite completes its life cycle in both humans and cats. It causes severe congenital abnormalities such as hydrocephalus and mental retardation in infants. Most T. gondii infections among humans occur by eating raw or undercooked meat containing T. gondii tissue cysts, by ingesting oocysts from soil), or by acquiring congenital infection through the placenta of mother.

Almost one third population of humans is infected worldwide by T. gondii [1]. Incidents of infection are ranging from lesser than $1 \%$ in younger persons in some places to $90 \%$ in older persons of some other areas and this is confirmed by serological studies. It is roughly estimated that $30 \%$ to $65 \%$ persons are infected worldwide by this parasite [2]. In the humans that have competent immune system this parasite infected central nervous system and this may be a factor of some psychiatric disorder. Instinctive abortions and some other life-threatening infections were caused by this parasite. Other than this, T. gondii is also responsible for personality disorder, dementia, encephalitis and anxiety [3].

Contact with contaminated oocytes is a major factor for infection in warm blooded animals which are found in cat feces (more than one day after shed), water and polluted soil. It is considered that beef is not a main reason of infection but undercooked infective meat is thinking to be a factor for infection [4]. Water is an important risk factor if untreated surface water is used. Farming with unsatisfied hygienic condition is also known a major risk factor. Unsatisfied personal habits and livestock handling, infrequent hand washing, unwashed kitchen knives/cutting boards after the cutting of fruits, raw meat and vegetables are the other risk factors for infection in human beings [5]. Moreover, because of developed personal hygienic condition and good handling of farm animal incidence of T. gondii has been reduced above the previous 20 years in animals whose meat may be used [2].
It is suggested that health education and public awareness should be provided to females, males and childrens to prevent infection. Most important health message for them is to avoid eating any meat that has not been properly cooked. Consequently, guidance needed to make sure that all fruits and vegetables are properly washed. Use of gloves should be done while contact with soil and working with animals should be eliminated. More studies should be conducted by using PCR to find out the prevalence of Toxoplasma gondii in food materials like meat, milk, eggs, fruits and vegetables.

\section{Bibliography}

1. Kijlstra A., et al. "Control of the risk of human toxoplasmosis transmitted by meat". International Journal of Parasitology 38 (2008): 1395-1370.

2. Tenter AM., et al. "Toxoplasma gondii: from animals to humans". International Journal of Parasitology 30 (2000): 1217-1258.

3. Roberts T., et al. "Economic losses caused by food borne parasitic diseases". Parasitology journal 10 (1994): 419-423.

4. Jones JL., et al. "Toxoplasma gondii infection in the United States. 1999-2004. Decline from the prior decade". American Journal of Tropical Medicine and Hygiene. 77 (2007): 405-410.

5. Bawm S., et al. "Serological survey and factors associated with Toxoplasm gondii infection in domestic goats in Myanmar". Scientifica 7 (2016): 92-97.

\section{Volume 2 Issue 8 August 2019 (C) All rights are reserved by Rafia Tabassum.}

